\title{
Classification of SD-OCT Volumes Using Local Binary Patterns: Experimental Validation for DME Detection
}

\author{
Guillaume Lemaître, ${ }^{1}$ Mojdeh Rastgoo, ${ }^{1}$ Joan Massich, ${ }^{1}$ Carol Y. Cheung, ${ }^{2}$ Tien Y. Wong, ${ }^{3}$ \\ Ecosse Lamoureux, ${ }^{3}$ Dan Milea, ${ }^{3}$ Fabrice Mériaudeau, ${ }^{1,4}$ and Désiré Sidibé ${ }^{1}$ \\ ${ }^{1}$ LE2I UMR6306, CNRS, Arts et Métiers, Université Bourgogne Franche-Comté, 12 rue de la Fonderie, 71200 Le Creusot, France \\ ${ }^{2}$ Department of Ophthalmology and Visual Sciences, The Chinese University of Hong Kong, Hong Kong Eye Hospital, \\ $147 \mathrm{~K}$ Argyle Street, Kowloon, Hong Kong \\ ${ }^{3}$ Singapore Eye Research Institute, Singapore National Eye Center, Singapore \\ ${ }^{4}$ Electrical \& Electronic Engineering Department, Center for Intelligent Signal and Imaging Research (CISIR), \\ Universiti Teknologi Petronas, Tronoh, 32610 Seri Iskandar, Perak, Malaysia
}

Correspondence should be addressed to Guillaume Lemaître; g.lemaitre58@gmail.com,

Mojdeh Rastgoo; mojdeh.rastgoo@gmail.com, and Joan Massich; mailsik@gmail.com

Received 27 November 2015; Revised 15 February 2016; Accepted 24 May 2016

Academic Editor: Theodore Leng

Copyright (C) 2016 Guillaume Lemaitre et al. This is an open access article distributed under the Creative Commons Attribution License, which permits unrestricted use, distribution, and reproduction in any medium, provided the original work is properly cited.

\begin{abstract}
This paper addresses the problem of automatic classification of Spectral Domain OCT (SD-OCT) data for automatic identification of patients with DME versus normal subjects. Optical Coherence Tomography (OCT) has been a valuable diagnostic tool for DME, which is among the most common causes of irreversible vision loss in individuals with diabetes. Here, a classification framework with five distinctive steps is proposed and we present an extensive study of each step. Our method considers combination of various preprocessing steps in conjunction with Local Binary Patterns (LBP) features and different mapping strategies. Using linear and nonlinear classifiers, we tested the developed framework on a balanced cohort of 32 patients. Experimental results show that the proposed method outperforms the previous studies by achieving a Sensitivity (SE) and a Specificity (SP) of $81.2 \%$ and $93.7 \%$, respectively. Our study concludes that the $3 \mathrm{D}$ features and high-level representation of $2 \mathrm{D}$ features using patches achieve the best results. However, the effects of preprocessing are inconsistent with different classifiers and feature configurations.
\end{abstract}

\section{Introduction}

Eye diseases such as Diabetic Retinopathy (DR) and Diabetic Macular Edema (DME) are the most common causes of irreversible vision loss in individuals with diabetes. Just in United States alone, health care and associated costs related to eye diseases are estimated at almost \$500 M [1]. Moreover, the prevalent cases of DR are expected to grow exponentially affecting over $300 \mathrm{M}$ people worldwide by 2025 [2]. Given this scenario, early detection and treatment of DR and DME play a major role in preventing adverse effects such as blindness. DME is characterized as an increase in retinal thickness within 1-disk diameter of the fovea center with or without hard exudates and sometimes associated with cysts [3]. Fundus images which have proven to be very useful in revealing most of the eye pathologies $[4,5]$ are not as good as OCT images which provide information about cross-sectional retinal morphology [6].

Many of the previous works on OCT image analysis have focused on the problem of retinal layers segmentation, which is a necessary step for retinal thickness measurements $[7,8]$. However, few have addressed the specific problem of DME and its associated features detection from OCT images. Figure 1 shows one normal B-scan and two abnormal B-scans.

A summary of the existing work can be found in Table 1. Srinivasan et al. [9] proposed a classification method to distinguish DME, Age-Related Macular Degeneration (AMD), and normal SD-OCT volumes. The OCT images are preprocessed by reducing the speckle noise by enhancing the sparsity in a transform-domain and flattening the retinal curvature to reduce the interpatient variations. Then, Histograms 


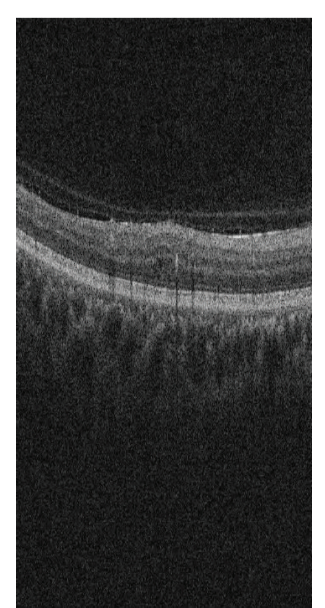

(a) Normal

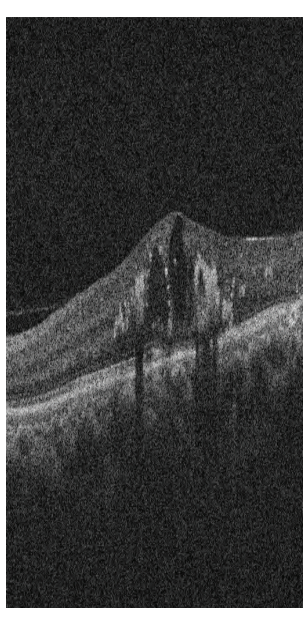

(b) DME-cyst

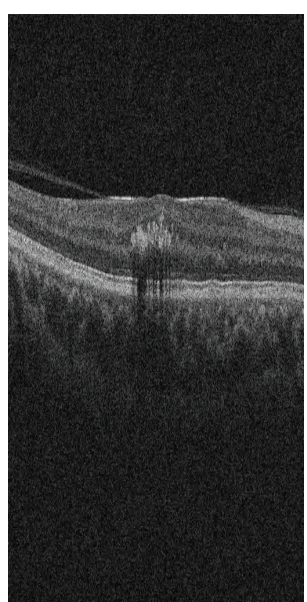

(c) DME-exudate

FIGURE 1: Example of SD-OCT images for normal (a) and DME patients (b)-(c) with cyst and exudate, respectively.

of Oriented Gradients (HOG) are extracted for each slice of a volume and linear Support Vector Machine (SVM) is used for classification. On a dataset of 45 patients equally subdivided into the three aforementioned classes, this method leads to a correct classification rate of $100 \%, 100 \%$, and $86.67 \%$ for normal, DME, and AMD patients, respectively. The images that have been used in their paper are publicly available but are already preprocessed (i.e., denoised), have different sizes for the OCT volumes, and do not offer a huge variability in terms of DME lesions, and some of them, without specifying which, have been excluded for the training phase; all these reasons prevent us from using this dataset to benchmark our work.

Venhuizen et al. proposed a method for OCT images classification using the Bag-of-Words (BoW) model [10]. The method starts with the detection and selection of key points in each individual B-scan, by keeping the most salient points corresponding to the top $3 \%$ of the vertical gradient values. Then, a texton of size $9 \times 9$ pixels is extracted around each key point, and Principal Component Analysis (PCA) is applied to reduce the dimension of every texton to get a feature vector of size 9. All extracted feature vectors are used to create a codebook using $k$-means clustering. Then, each OCT volume is represented in terms of this codebook and is characterized as a histogram that captures the codebook occurrences. These histograms are used as feature vector to train a Random Forest (RF) with a maximum of 100 trees. The method was used to classify OCT volumes between AMD and normal cases and achieved an Area Under the Curve (AUC) of 0.984 with a dataset of 384 OCT volumes.

Liu et al. proposed a methodology for detecting macular pathology in OCT images using LBP and gradient information as attributes [11]. The method starts by aligning and flattening the images and creating a 3-level multiscale spatial pyramid. The edge and LBP histograms are then extracted from each block of every level of the pyramid. All the obtained histograms are concatenated into a global descriptor whose dimensions are reduced using PCA. Finally a SVM with a Radial Basis Function (RBF) kernel is used as classifier. The method achieved good results in detection OCT scan containing different pathologies such as DME or AMD, with an AUC of 0.93 using a dataset of 326 OCT scans.

Lemaitre et al. [12] proposed using 2D and 3D LBP features extracted from denoised volumes and dictionary learning using the BoW models [13]. In the proposed method all the dictionaries are learned with the same size of "visual words" $(k=32)$ and final descriptors are classified using RF classifier.

The work described in this paper is an extension of our previous work [12]. In this research, beside the comparison of $2 \mathrm{D}$ and $3 \mathrm{D}$ features, we explore different possible representations of the features and different preprocessing steps for OCT data (i.e., aligning, flattening, and denoising). We also compare the performances of different classifiers.

This paper is organized as follows: the proposed framework is explained in Section 2, while the experiments and results are discussed through Sections 3 and 4. Finally, the conclusion and avenue for future directions are drawn in Section 5.

\section{Materials and Methods}

The proposed method, as well as its experimental setup, for OCT volume classification is outlined in Figure 2. The methodology is formulated as a standard classification procedure which consists of five steps. First, the OCT volumes are preprocessed as presented in detail in Section 2.1. Then, LBP and LBP-TOP features are detected, mapped, and represented as discussed in depth in Sections 2.2, 2.3, and 2.4, respectively. Finally, the classification step is presented in Section 2.5.

2.1. Image Preprocessing. This section describes the set of preprocessing techniques which aim at enhancing the OCT volume. The influences of these preprocessing methods and their possible combinations are extensively studied in Section 3.

2.1.1. Non-Local Means (NLM). OCT images suffer from speckle noise, like other image modalities such as Ultrasound 


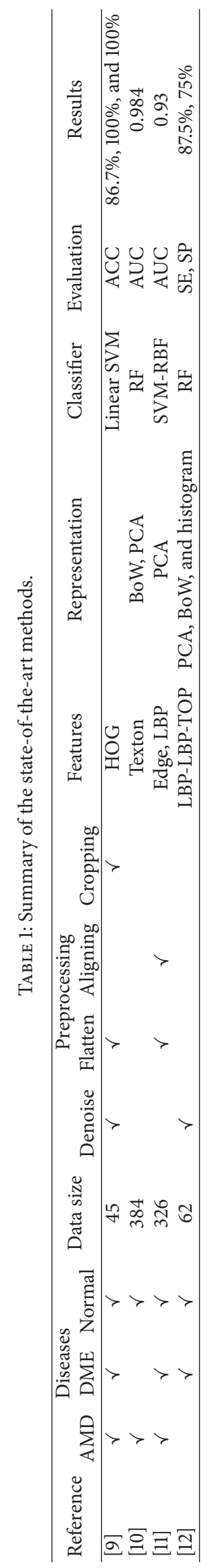




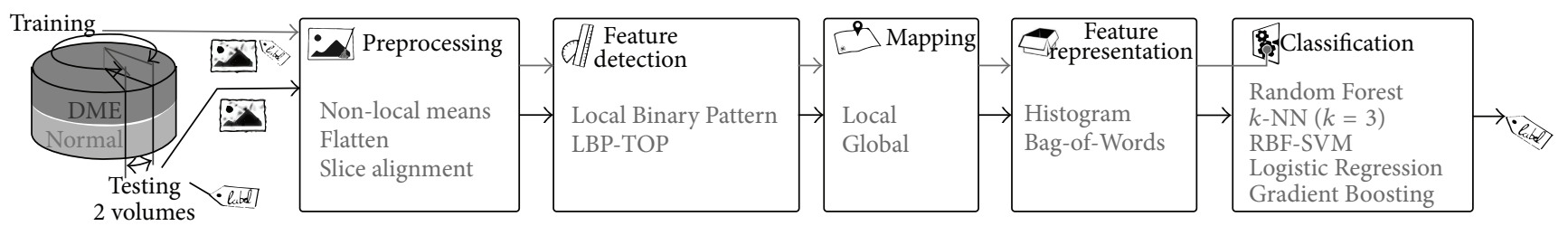

FIGURE 2: Our proposed classification pipeline.

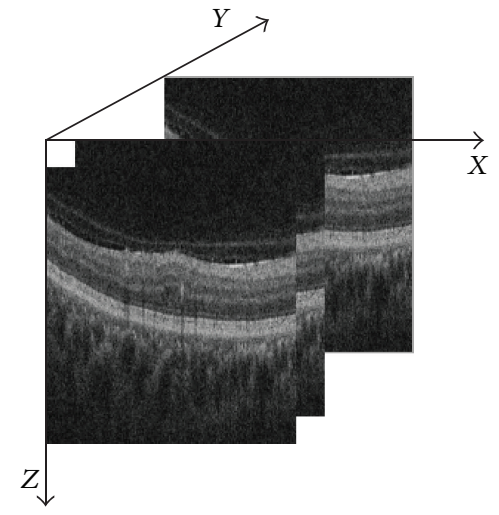

(a)

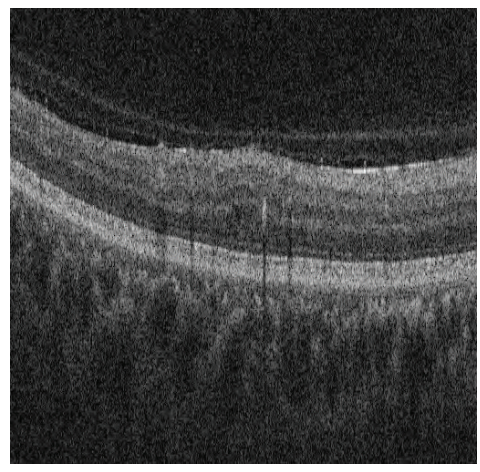

(b)

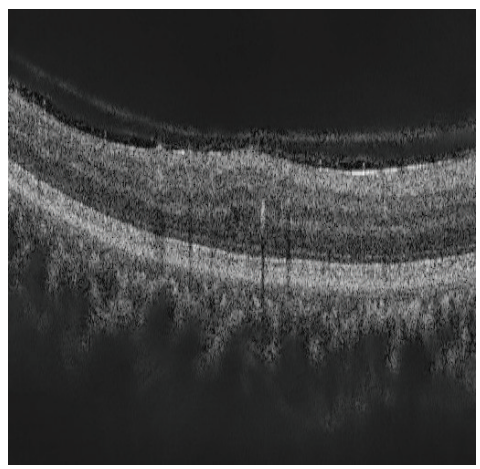

(c)

FIGURE 3: OCT: (a) organization of the OCT data, (b) original image, and (c) NLM filtering. Note that the images have been negated for visualization purposes.

(US) [14]. The OCT volumes are enhanced by denoising each B-scan (i.e., each $(x-z)$ slice) using the NLM [15], as shown in Figure 3. NLM has been successfully applied to US images to reduce speckle noise and outperforms other common denoising methods [16]. NLM filtering preserves fine structures as well as flat zones, by using all the possible self-predictions that the image can provide rather than local or frequency filters such as Gaussian, anisotropic, or Wiener filters [15].

2.1.2. Flattening. Textural descriptors characterize spatial arrangement of intensities. However, the OCT scans suffer from large type of variations: inclination angles, positioning, and natural curvature of the retina [11]. Therefore, these variations have to be taken into account to ensure a consistent characterization of the tissue disposition, regardless of the location in the retina. This invariance can be achieved in different manners: (i) using a rotation invariant descriptor (cf. Section 2.2) or (ii) unfolding the curvature of the retina. This latter correction is known as image flattening which theoretically consists of two distinct steps: (i) estimate and fit the curvature of the Retinal Pigment Epithelium (RPE) and (ii) warp the OCT volume such that the RPE becomes flat.

Our correction is similar to the one of Liu et al. [11]: each B-scan is thresholded using Otsu's method followed by a median filtering to detect the different retina layers (see Figures 4(c) and 4(d)). Then, a morphological closing and opening is applied to fill the holes and the resulting area is fitted using a second-order polynomial (see Figure 4(d)). Finally, the scan is warped such that the curve becomes a line as presented in Figures 4(e) and 4(f).
2.1.3. Slice Alignment. The flattening correction does not enforce an alignment through the OCT volume. Thus, in addition to the flattening correction, the warped curves of each B-scan are positioned at the same altitude in the $z$-axis.

2.2. Feature Detection. In this research, we choose to detect simple and efficient LBP texture features with regard to each OCT slice and volume. LBP is a texture descriptor based on the signs of the differences of a central pixel with respect to its neighboring pixels [17]. These differences are encoded in terms of binary patterns as follows:

$$
\begin{gathered}
\operatorname{LBP}_{P, R}=\sum_{p=0}^{P-1} s\left(g_{p}-g_{c}\right) 2^{p}, \\
s(x)= \begin{cases}1 & \text { if } x \geq 0 \\
0 & \text { otherwise }\end{cases}
\end{gathered}
$$

where $g_{c}, g_{p}$ are the intensities of the central pixel and a given neighbor pixel, respectively, and $P$ is the number of sampling points in the circle of radius $R$.

Ojala et al. further extended the original LBP formulation to achieve rotation invariance at the expense of limiting the texture description to the notion of circular "uniformity" [17]. Referring to the coordinate system defined in Figure 3(a), the LBP codes are computed on each $(x-z)$ slice, leading to a set of LBP maps, a map for each $(x-z)$ slice.

Volume encoding is later proposed by Zhao et al. by computing LBP descriptors in three orthogonal planes, so-called LBP-TOP [18]. More precisely, the LBP codes are computed 


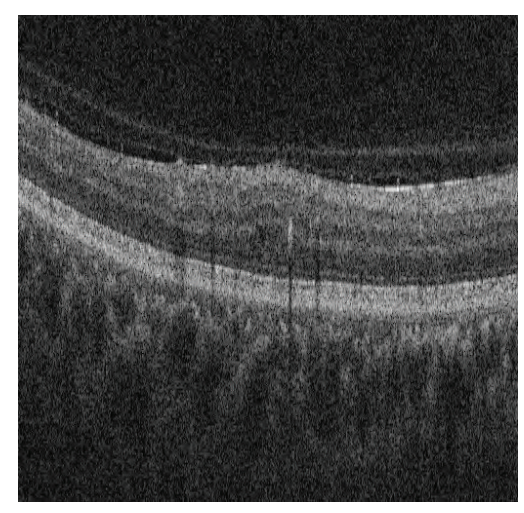

(a)

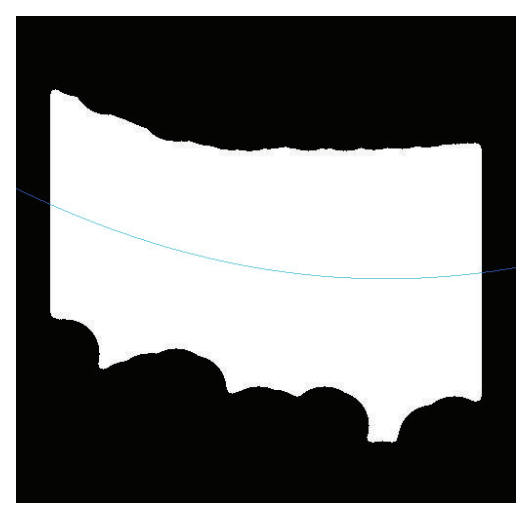

(d)

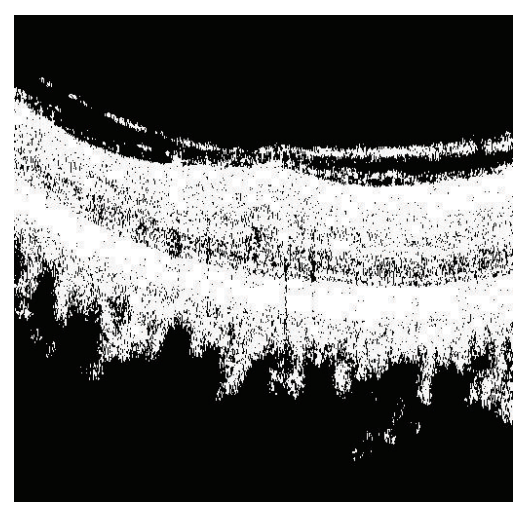

(b)

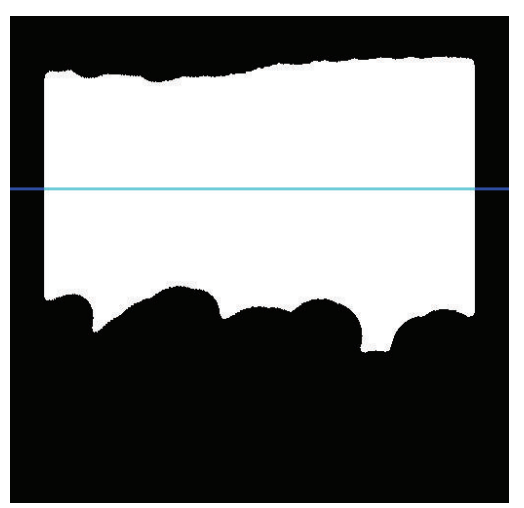

(e)

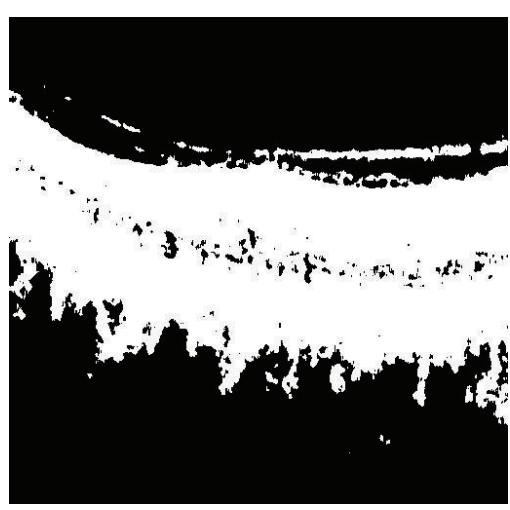

(c)

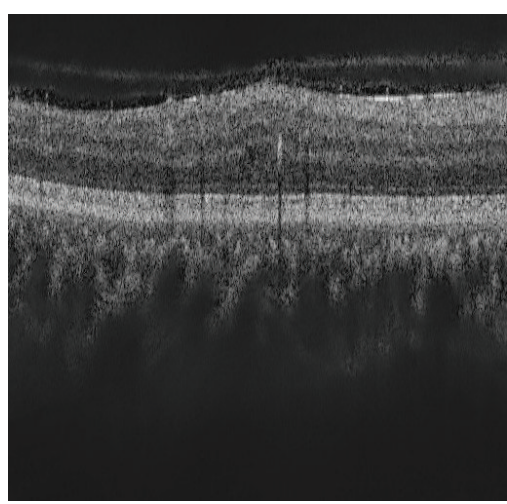

(f)

FIGURE 4: Flattening procedure: (a) original image, (b) thresholding, (c) median filtering, (d) curve fitting, (e) warping, and (f) flatten image.

TABLE 2: Number of patterns ( $\left.\mathrm{LBP}_{\text {\#pat }}\right)$ for different sampling points and radius $(\{P, R\})$ of the LBP descriptor.

\begin{tabular}{lccc}
\hline \multicolumn{4}{c}{ Sampling point for a radius $(\{P, R\})$} \\
\hline & $\{8,1\}$ & $\{16,2\}$ & $\{24,3\}$ \\
$\mathrm{LBP}_{\text {\#pat }}$ & 10 & 18 & 26 \\
\hline
\end{tabular}

considering the $(x-z)$ plane, $(x-y)$ plane, and $(y-z)$ plane, independently. Thus, three sets of LBP maps are obtained, one for each orthogonal plane.

In this work, we consider rotation invariant and uniform LBP and LBP-TOP features with various sampling points (i.e., $\{8,16,24\})$ with respect to different radius (i.e., $\{1,2,3\})$. The number of patterns $\left(\mathrm{LBP}_{\# \text { pat }}\right)$ in regard to each configuration is reported in Table 2.

2.3. Mapping. The mapping stage is used to partition the previously computed LBP maps; for this work, two mapping strategies are defined: (i) global and (ii) local mapping. The size of the feature descriptor is summarized in Table 3.

2.3.1. Global. Global mapping extracts the final descriptors from the 2D feature image for LBP and 3D volume for LBPTOP. Therefore, for a volume with $d$ slices, the global-LBP mapping will lead to the extraction of $d$ elements, while
TABLE 3: Size of a descriptor for an SD-OCT volume. $d$ denotes the number of slices in the volume, $N$ the number of $2 \mathrm{D}$ windows, and $N^{\prime}$ the number of 3D subvolumes, respectively.

\begin{tabular}{lcc}
\hline & Global mapping & Local mapping \\
\hline LBP & $d \times \mathrm{LBP}_{\text {\#pat }}$ & $(N \times d) \times \mathrm{LBP}_{\text {\#pat }}$ \\
LBP-TOP & $1 \times\left(3 \times \mathrm{LBP}_{\text {\#pat }}\right)$ & $N^{\prime} \times\left(3 \times \mathrm{LBP}_{\text {\#pat }}\right)$ \\
\hline
\end{tabular}

the global-LBP-TOP represents the whole volume as a single element. The global mapping for 2D images and 3D volume is shown in Figures 5(a) and 5(b).

2.3.2. Local. Local mapping extracts the final descriptors from a set of $(m \times m)$ 2D patches for LBP and a set of $(m \times$ $m \times m)$ subvolumes for LBP-TOP. Given $N$ and $N^{\prime}$ as the total number of $2 \mathrm{D}$ patches and 3D subvolumes, respectively, the local-LBP approach provides $N \times d$ elements, while localLBP-TOP provides $N^{\prime}$ elements. This mapping is illustrated in Figures 5(c) and 5(d).

2.4. Feature Representation. Two strategies are used to describe each OCT volume's texture.

2.4.1. Low-Level Representation. The texture descriptor of an OCT volume is defined as the concatenation of the LBP 


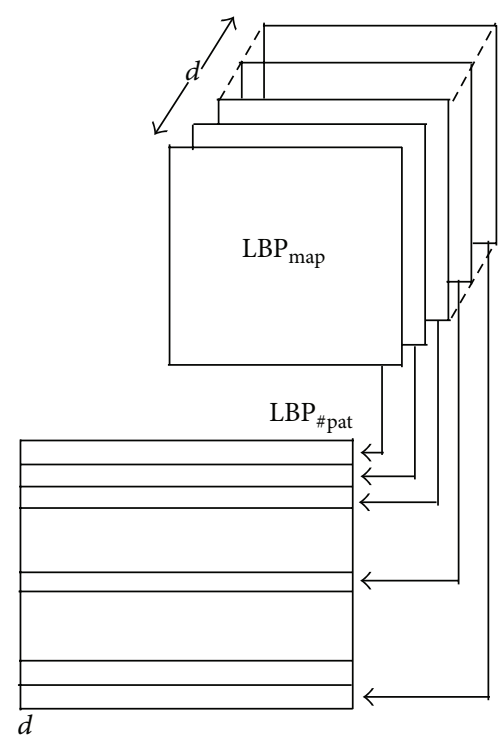

(a)

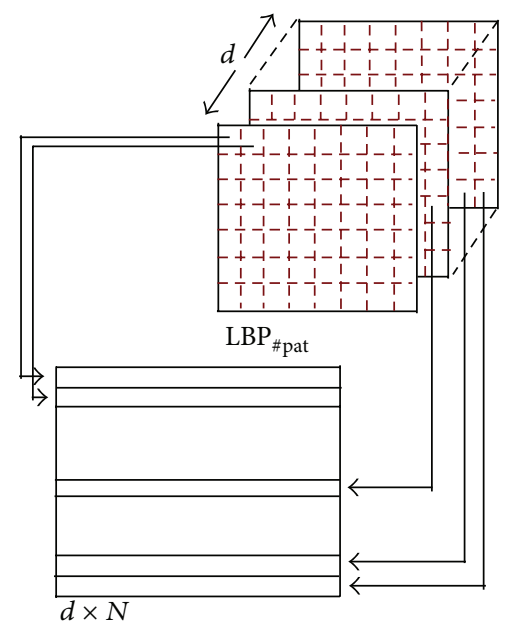

(c)

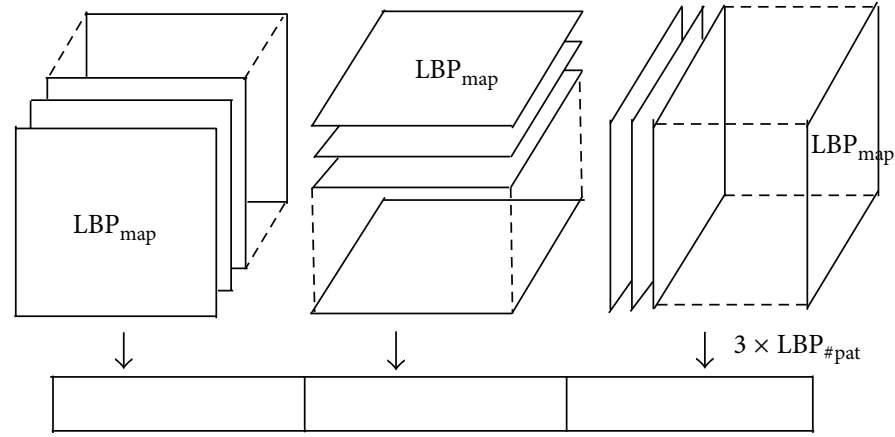

(b)

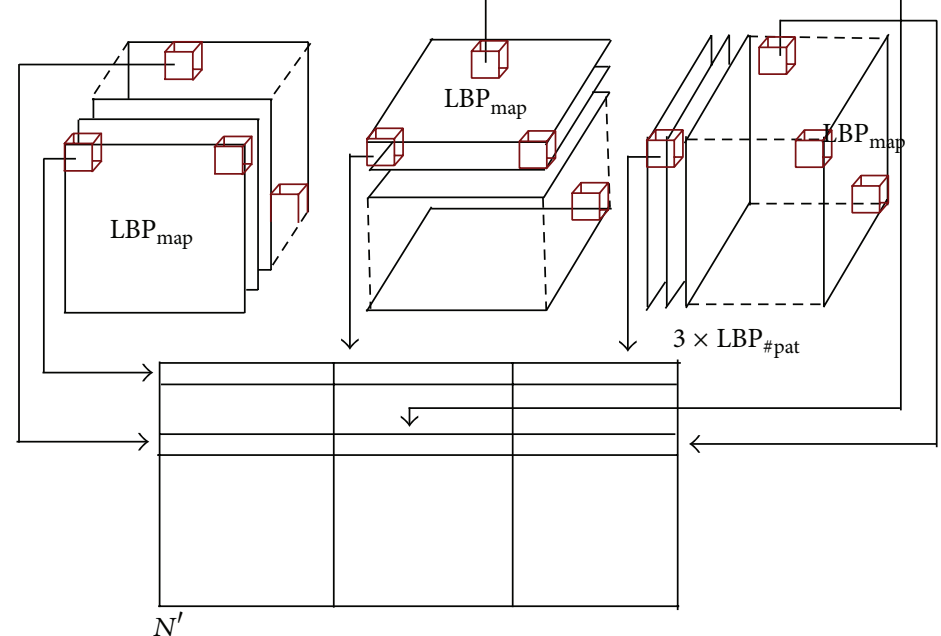

(d)

FIGURE 5: Graphical representation of the feature extraction: (a) extraction of LBP for global mapping, (b) extraction of LBP-TOP for global mapping, (c) extraction of LBP for local mapping, and (d) extraction of LBP-TOP for local mapping.

histograms with the global mapping. The LBP histograms are extracted from the previously computed LBP maps (see Section 2.2). Therefore, the LBP-TOP final descriptor is computed through the concatenation of the LBP histograms of the three orthogonal planes with the final size of $3 \times$ $\mathrm{LBP}_{\text {\#pat }}$. More precisely, an LBP histogram is computed for each set of LBP maps $(x-z)$ plane, $(x-y)$ plane, and $(y-z)$ plane, respectively. Similarly, the LBP descriptor is defined through concatenation of the LBP histograms per each $(x-z)$ slice with the final size of $d \times \mathrm{LBP}_{\text {\#pat }}$.

2.4.2. High-Level Representation. The concatenation of histograms employed in the low-level representation in conjunction with either global or local mapping can lead to a highdimensional feature space. For instance, local mapping results in a size of $N \times d \times \mathrm{LBP}_{\# \text { pat }}$ for the final LBP descriptor and
$N^{\prime} \times \mathrm{LBP}_{\# \text { pat }}$ for the final LBP-TOP descriptor, where $N$ and $N^{\prime}$ are the total number of 2D patches and 3D subvolumes, respectively. High-level representation simplifies this highdimensional feature space into a more discriminant lower space. BoW approach is used for this purpose [13]. This model represents the features by creating a codebook or visual dictionary, from the set of low-level features. The set of low-level features are clustered using $k$-means to create the codebook with $k$ clusters or visual words. After creating the codebook from the training set, the low-level descriptors are replaced by their closest word within the codebook. The final descriptor is a histogram of size $k$ which represents the codebook occurrences for a given mapping.

2.5. Classification. The last step of our framework consists in the classification of SD-OCT volumes as normal or DME. 
For that matter, five different classifiers are used: (i) $k$-Nearest Neighbor (NN), (ii) Logistic Regression (LR) [19], (iii) Random Forest (RF) [20], (iv) Gradient Boosting (GB) $[21,22]$, and (v) Support Vector Machines (SVM) [23, 24]. Details regarding the parameters used in our experiments are provided in Section 3.

\section{Experiments}

A set of three experiments is designed to test the influence of the different blocks of the proposed framework in comparison to our previous work [12]. These experiments are designed as follows:

(i) Experiment 1 evaluates the effects of number of words used in BoW (high-level representation).

(ii) Experiment 2 evaluates the effects of different preprocessing steps and classifiers on high-level representation.

(iii) Experiment 3 evaluates the effects of different preprocessing steps and classifiers on low-level representation.

Table 4 reports the experiments which have been carried out in [12] as a baseline and outlines the complementary experimentation here proposed. The reminder of this section details the common configuration parameters across the experiments, while the detailed explanations are presented in the following subsections.

All the experiments are performed using a private dataset (see Section 3.1) and are reported as presented in Section 3.2. In all the experiments, LBP and LBP-TOP features are extracted using both local and global mapping for different sampling points of 8,16 , and 24 for radius of 1,2 , and 3 pixels, respectively. The partitioning for local-mapping is set to $(7 \times$ 7)-pixel patch for $2 \mathrm{D}$ LBP and $(7 \times 7 \times 7)$-pixel subvolume for LBP-TOP.

3.1. SERI Dataset. This dataset was acquired by the Singapore Eye Research Institute (SERI), using CIRRUS ${ }^{\text {mi }}$ (Carl Zeiss Meditec, Inc., Dublin, CA) SD-OCT device. The dataset consists of 32 OCT volumes (16 DME and 16 normal cases). Each volume contains 128 B-scan with resolution of $512 \times 1024$ pixels. All SD-OCT images are read and assessed by trained graders and identified as normal or DME cases based on evaluation of retinal thickening, hard exudates, intraretinal cystoid space formation, and subretinal fluid.

3.2. Validation. All the experiments are evaluated in terms of Sensitivity (SE) and Specificity (SP) using the LOPO-CV strategy, in line with [12]. SE and SP are statistics driven from the confusion matrix as depicted in Figure 6. The SE evaluates the performance of the classifier with respect to the positive class, while the SP evaluates its performance with respect to negative class. The use of LOPO-CV implies that, at each round, a pair of DME-normal volumes is selected for testing while the remaining volumes are used for training. Subsequently, no SE or SP variance can be reported. However,

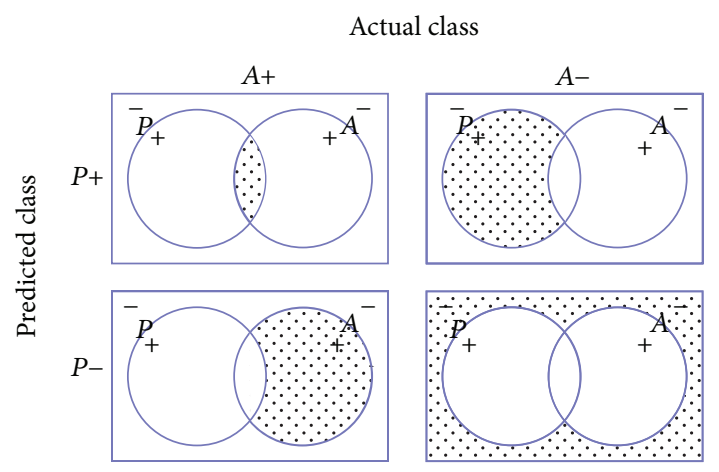

(a) Confusion matrix with truly and falsely positive samples detected (TP, FP) in the first row, from left to right, and the falsely and truly negative samples detected (FN, TN) in the second row, from left to right

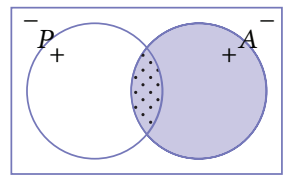

$$
\mathrm{SE}=\frac{\mathrm{TP}}{\mathrm{TP}+\mathrm{FN}}
$$

(b) SE and SP evaluation, corresponding to the ratio of the dotted area to the blue area

FIGURE 6: Evaluation metrics: (a) confusion matrix and (b) SE-SP.

LOPO-CV strategy has been adopted despite this limitation due to the reduced size of the dataset.

3.3. Experiment 1. This experiment intends to find the optimal number of words and its effect on the different configurations (i.e., preprocessing and feature representation), on the contrary to [12], where the codebook size was arbitrarily set to $k=32$.

Several preprocessing strategies are used: (i) NLM, (ii) a combination of NLM and flattening (NLM+F), and (iii) a combination of NLM, flattening, and aligning $(\mathrm{NLM}+\mathrm{F}+\mathrm{A})$. LBP and LBP-TOP descriptors are detected using the default configuration. Volumes are represented using BoW, where the codebook size ranges within $k \in\{10,20,30, \ldots, 100,200$, $\ldots, 500,1000\}$. Finally, the volumes are classified using LR. The choice of this linear classifier avoids the case that the results get boosted by the classifier. In this manner, any improvement would be linked to the preprocessing and the size of the codebook.

The usual build of the codebook consists of clustering the samples in the feature space using $k$-means (see Section 2.4). However, this operation is rather computationally expensive and the convergence of the $k$-means algorithm for all codebook sizes is not granted. Nonetheless, Nowak et al. [25] pointed out that randomly generated codebooks can be used at the expense of accuracy. Thus, the codebook is randomly generated since the final aim is to assess the influence of the codebook size and not the performance of the framework. For this experiment, the codebook building is carried out using random initialization using $k$-means++ algorithm [26], which is usually used as a $k$-means initialization algorithm. 


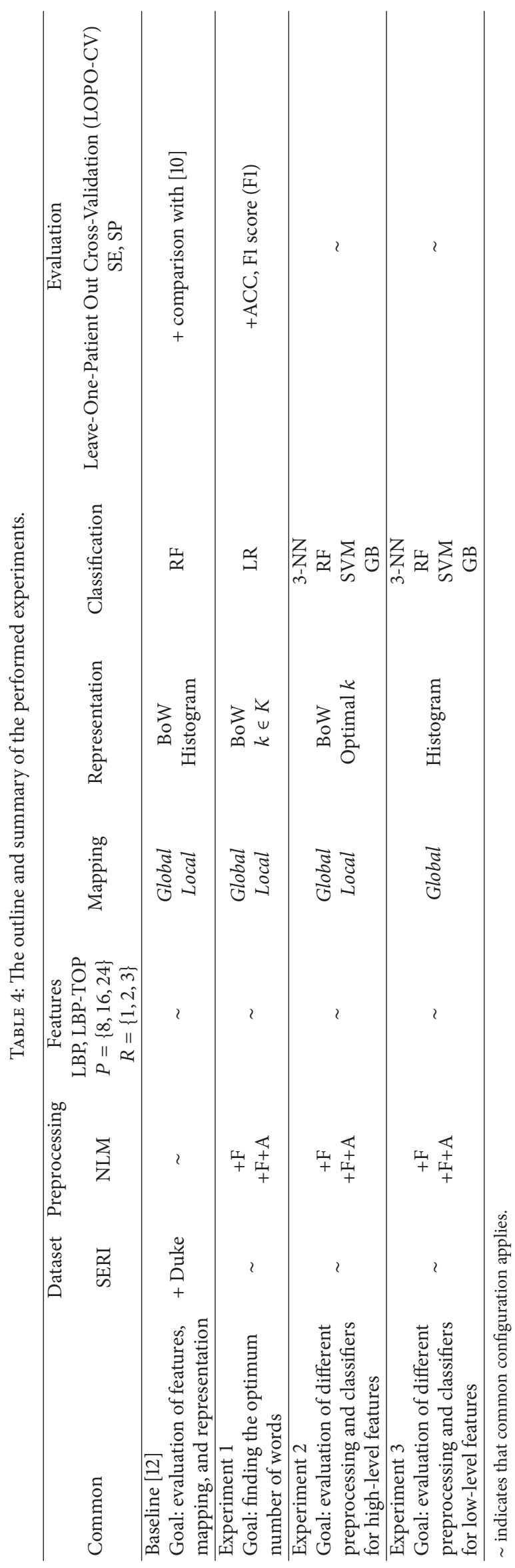


TABLE 5: Summary of all the results in descending order.

\begin{tabular}{|c|c|c|c|c|c|c|c|c|c|c|c|c|}
\hline \multirow{2}{*}{ Line } & \multirow{2}{*}{ Experiment } & \multicolumn{2}{|c|}{ Evaluation } & \multirow{2}{*}{ Pre-processing } & \multicolumn{4}{|c|}{ Feat. Detection } & \multirow{2}{*}{ Mapping } & \multirow{2}{*}{ Feat. Representation } & \multirow{2}{*}{ Classifier } & \multirow{2}{*}{ BoW } \\
\hline & & SE & SP & & Type & $\{8,1\}$ & $\{16,2\}$ & $\{24,3\}$ & & & & \\
\hline 1 & 2 & 81.2 & 93.7 & $\mathrm{NLM}+\mathrm{F}$ & LBP & $r$ & & & Local & High & SVM & $\checkmark$ \\
\hline 2 & 2 & 75.0 & 93.7 & $\mathrm{NLM}+\mathrm{F}+\mathrm{A}$ & LBP & $\checkmark$ & & & Local & High & SVM & $\checkmark$ \\
\hline 3 & 2 & 75.0 & 93.7 & NLM & LBP & $\checkmark$ & & & Local & High & SVM & $\checkmark$ \\
\hline 4 & 2 & 75.0 & 100 & NLM & LBP-TOP & & $\checkmark$ & & Local & High & SVM & $\checkmark$ \\
\hline 5 & 2 & 81.2 & 87.5 & NLM & LBP-TOP & $\checkmark$ & & & Local & High & SVM & $\checkmark$ \\
\hline 6 & 2 & 81.2 & 87.5 & $\mathrm{NLM}+\mathrm{F}+\mathrm{A}$ & LBP-TOP & & $\checkmark$ & & Local & High & $\mathrm{RF}$ & $\checkmark$ \\
\hline 7 & 2 & 81.2 & 81.2 & NLM & LBP & $\checkmark$ & & & Local & High & $\mathrm{RF}$ & $\checkmark$ \\
\hline 8 & 3 & 81.2 & 81.2 & NLM & LBP-TOP & & & $\checkmark$ & Global & Low & $\mathrm{RF}$ & \\
\hline 9 & 2 & 81.2 & 81.2 & $\mathrm{NLM}+\mathrm{F}$ & LBP-TOP & $\checkmark$ & & & Local & High & SVM & $\checkmark$ \\
\hline 10 & 3 & 81.2 & 81.2 & $\mathrm{NLM}+\mathrm{F}+\mathrm{A}$ & LBP-TOP & & & $\checkmark$ & Global & Low & GB & \\
\hline 11 & 3 & 81.2 & 81.2 & NLM+F & LBP-TOP & & & $\checkmark$ & Global & Low & $\mathrm{RF}$ & \\
\hline 12 & 2 & 75.0 & 87.5 & NLM & LBP & $\checkmark$ & & & Local & High & $k-\mathrm{NN}$ & $\checkmark$ \\
\hline 13 & Lemaitre et al. [12] & 75.0 & 87.5 & NLM & LBP & $\checkmark$ & & & Local & High & $\mathrm{RF}$ & $\checkmark$ \\
\hline 14 & Lemaitre et al. [12] & 75.0 & 87.5 & NLM & LBP-TOP & & $\checkmark$ & & Global & Low & $\mathrm{RF}$ & \\
\hline 15 & 2 & 68.7 & 93.7 & NLM & LBP & $\checkmark$ & & & Global & High & $\mathrm{RF}$ & $\checkmark$ \\
\hline 16 & 3 & 75 & 81.2 & $\mathrm{NLM}+\mathrm{F}+\mathrm{A}$ & LBP-TOP & & & $\checkmark$ & Global & Low & $\mathrm{RF}$ & \\
\hline 17 & 2 & 68.7 & 81.2 & NLM & LBP-TOP & & $\checkmark$ & & Local & High & $\mathrm{RF}$ & $\checkmark$ \\
\hline 18 & 3 & 62.5 & 93.7 & NLM & LBP-TOP & & $\checkmark$ & & Global & Low & SVM & \\
\hline 19 & 3 & 68.7 & 87.5 & NLM & LBP-TOP & & $\checkmark$ & & Global & Low & $\mathrm{RF}$ & \\
\hline 20 & 3 & 68.7 & 81.2 & NLM & LBP-TOP & & & & Global & Low & $\mathrm{RF}$ & \\
\hline 21 & 3 & 75.0 & 75.0 & NLM & LBP-TOP & & & & Global & Low & $\mathrm{RF}$ & \\
\hline 22 & 3 & 68.7 & 75.0 & $\mathrm{NLM}+\mathrm{F}$ & LBP-TOP & $\checkmark$ & & & Global & Low & SVM & \\
\hline 23 & 3 & 56.2 & 75.0 & NLM & LBP & & & $\checkmark$ & Global & Low & $\mathrm{RF}$ & \\
\hline 24 & 3 & 56.2 & 75.0 & $\mathrm{NLM}+\mathrm{F}$ & LBP & & $\checkmark$ & & Global & Low & $k-\mathrm{NN}$ & \\
\hline 25 & 3 & 56.2 & 75.0 & $\mathrm{NLM}+\mathrm{F}+\mathrm{A}$ & LBP & & $\checkmark$ & & Global & Low & $k-\mathrm{NN}$ & \\
\hline 26 & Venhuizen et al. [10] & 61.5 & 58.8 & & & & & & & & & \\
\hline
\end{tabular}

For this experiment, SE and SP are complemented with ACC and F1 score (see (2)). ACC offers an overall sense of the classifier performance, and F1 illustrates the trade-off between SE and precision. Precision or positive predictive value is a measure of algorithm exactness and is defined as a ratio of True Positive over the total predicted positive samples:

$$
\begin{aligned}
\mathrm{ACC} & =\frac{\mathrm{TP}+\mathrm{TN}}{\mathrm{TP}+\mathrm{TN}+\mathrm{FP}+\mathrm{FN}}, \\
\mathrm{F} 1 & =\frac{2 \mathrm{TP}}{2 \mathrm{TP}+\mathrm{FP}+\mathrm{FN}} .
\end{aligned}
$$

Table 6 in Appendix shows the results obtained for the optimal dictionary size while the complete set of all ACC and F1 graphics can be found at [27]. According to the obtained results, it is observed that the optimum number of words is smaller for local-LBP features in comparison to local-LBPTOP and global-LBP, respectively. Using LR classifier, the best performances were achieved using local-LBP with 70 words (SE and SP of 75.0\%) and local-LBP-TOP with 500 words (SE and SP of $75.0 \%$ as well). These results are shown in bold in Table 6 in Appendix.

3.4. Experiment 2. This experiment explores the improvement associated with (i) different preprocessing methods and (ii) using larger range of classifiers (i.e., linear and nonlinear) on the high-level representation.

All the preprocessing stages are evaluated (NLM, NLM+F, and $\mathrm{NLM}+\mathrm{F}+\mathrm{A})$. In this experiment, the codebooks for the BoW representation of LBP and LBP-TOP features are computed using regular $k$-means algorithm which is initialized using $k$-means++, where $k$ is chosen according to the findings of Experiment 1. Finally, the volumes are classified using $k$ $\mathrm{NN}, \mathrm{RF}, \mathrm{GB}$, and SVM. The $k$-NN classifier is used in conjunction with the 3 nearest neighbors rule to classify the test set. The RF and GB classifiers are trained using 100 unpruned trees, while SVM classifier is trained using an RBF kernel and its parameters $C$ and $\gamma$ are optimized through grid-search.

Complete list of the obtained results from this experiment is shown in Table 7 in Appendix. Despite the fact that highest performances are achieved when $\mathrm{NLM}+\mathrm{F}$ or $\mathrm{NLM}+\mathrm{F}+\mathrm{A}$ is used, most configurations decline when applied with extra preprocessing stages. The best results are achieved using SVM followed by RF.

3.5. Experiment 3. This experiment replicates Experiment 2 for the case of low-level representation of LBP and LBP-TOP features extracted using global mapping.

The obtained results from this experiment are listed in Table 8 in Appendix. In this experiment, flattening the Bscan boosts the results of the best performing configuration. 


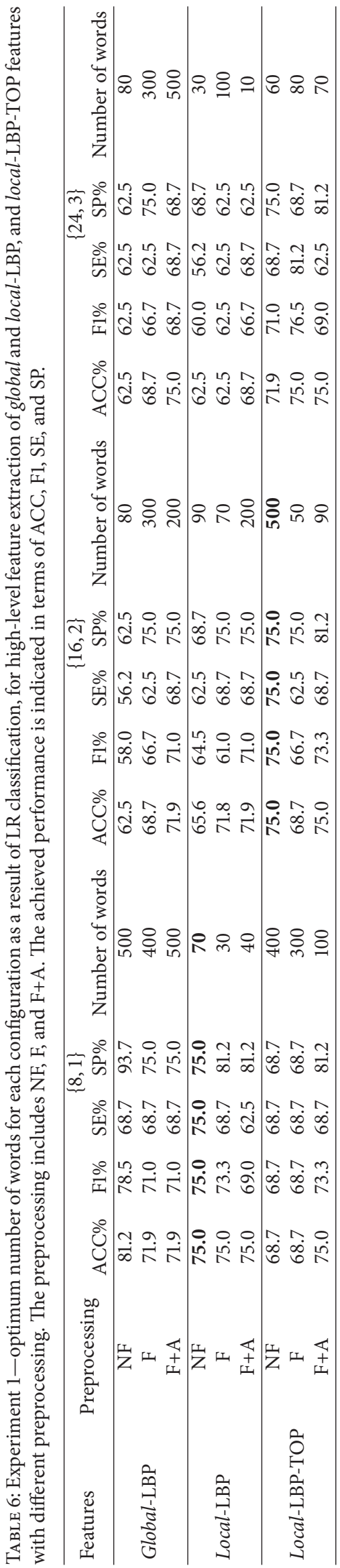




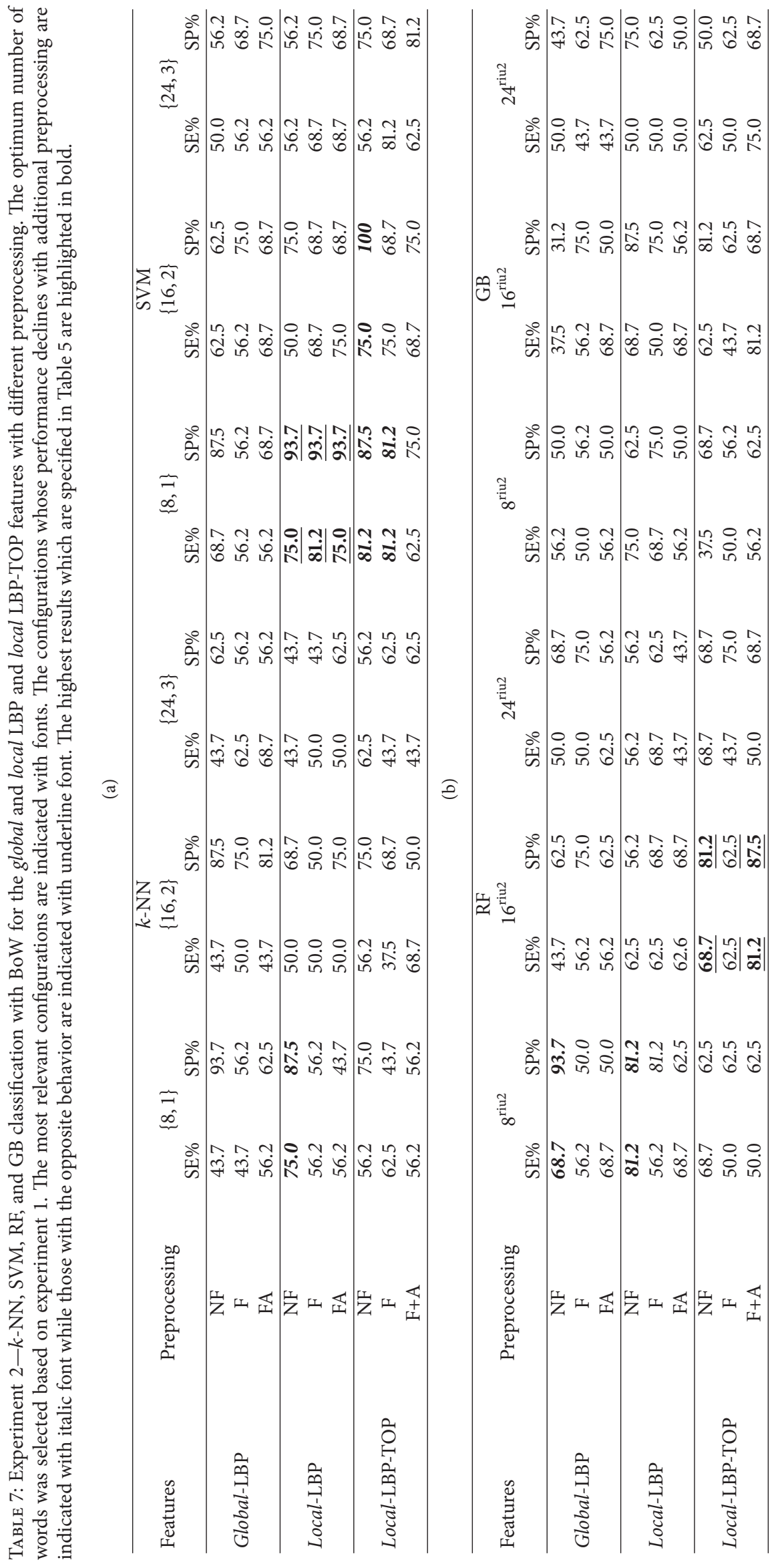




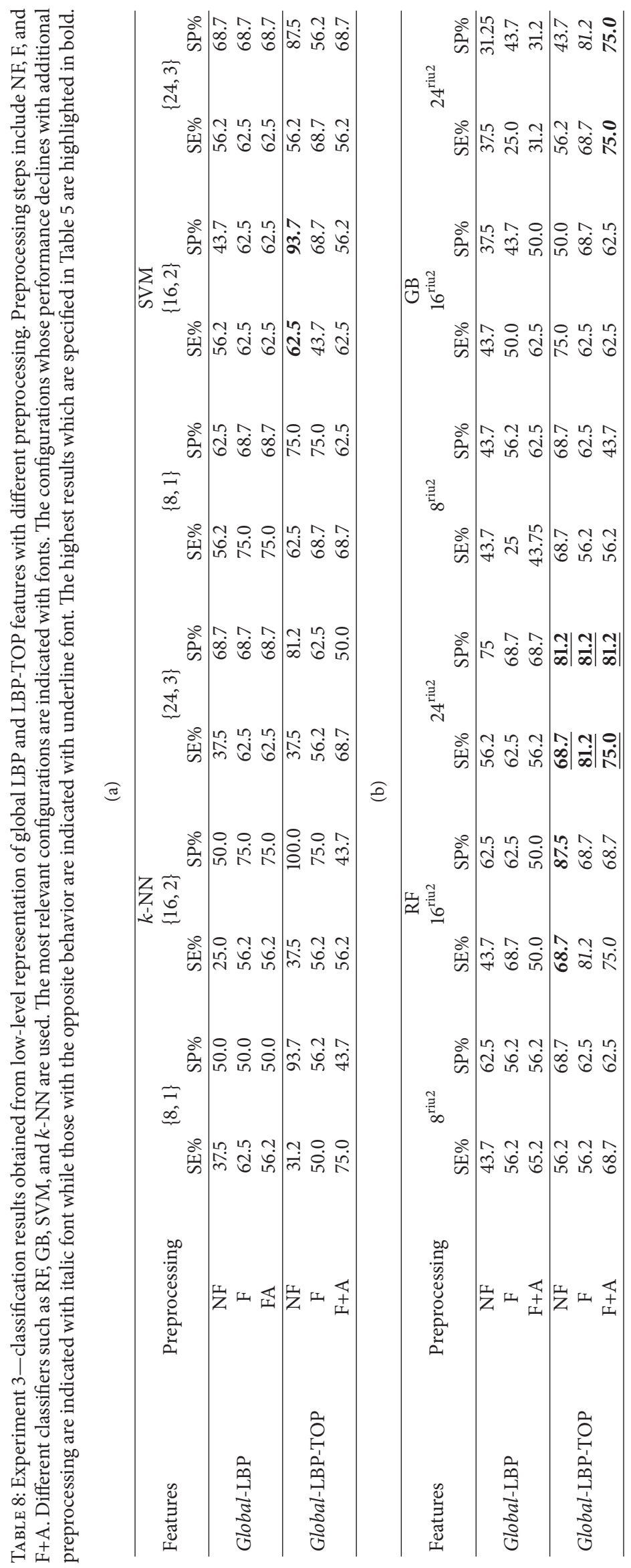


However, its effects is not consistent across all the configurations. RF has a better performance by achieving better SE $(81.2 \%, 75.0 \%$, and $68.7 \%)$, while SVM achieves the highest SP (93.7\%), see Table 8 in the Appendix.

In terms of classifier, RF has a better performance than the others despite the fact that the highest SP is achieved using SVM.

\section{Results and Discussion}

Table 5 combines the obtained results from Section 3 with those reported by Lemaitre et al. [12], while detailing the frameworks configurations. This table shows the achieved performances with SE higher than $55 \%$.

The obtained results indicate that expansion and tuning of our previous framework improve the results. Tuning the codebook size, based on the finding of Experiment 1, leads to an improvement of $6 \%$ in terms of SE (see Table 5 at lines 7 and 13). Furthermore, the fine-tuning of our framework (see Section 2) also leads to an improvement of $6 \%$ in both SE and SP (see Table 5 at lines 1 and 13). Our framework also outperforms the proposed method of [10] with an improvement of $20 \%$ and $36 \%$ in terms of SE and SP, respectively.

Note that although the effects of preprocessing are not consistent through all the performances, the best results are achieved with $\mathrm{NLM}+\mathrm{F}$ and $\mathrm{NLM}+\mathrm{F}+\mathrm{A}$ configurations as preprocessing stages. In general, the configurations presented in Experiment 2 outperform the others, in particular the highlevel representation of locally mapped features with an SVM classifier. Focusing on the most desirable radius and sampling point configuration, smaller radius and sampling points are more effective in conjunction with local mapping, while global mapping benefits from larger radius and sampling points.

\section{Conclusions}

The work presented here addresses automatic classification of SD-OCT volumes as normal or DME. In this regard, an extensive study is carried out covering the (i) effects of different preprocessing steps, (ii) influence of different mapping and feature extraction strategies, (iii) impact of the codebook size in BoW, and (iv) comparison of different classification strategies.

While outperforming the previous studies [10, 12], the obtained results in this research showed the impact and importance of optimal codebook size, the potential of 3D features, and high-level representation of 2D features while extracting from local patches.

The strengths of SVM while being used along with BoW approach and RF classifier while being used with global mapping were shown. In terms of preprocessing steps, although the highest performances are achieved while alignment and flattening were used in the preprocessing, it was shown that the effects of these extra steps are not consistent for all the cases and do not guarantee a better performance.

Several avenues for future directions can be explored. The flattening method proposed by Liu et al. flattens roughly the
RPE due to the fact that the RPE is not segmented. Thus, in order to have a more accurate flattening preprocessing, the RPE layer should be presegmented as proposed by Garvin et al. [28]. In this work, the LBP invariant to rotation was used and the number of patterns encoded is reduced. Once the data are flattened, the nonrotation invariant LBP could be studied since this descriptor encodes more patterns. In addition to LBP, other feature descriptors can be included in the framework.

\section{Appendix}

\section{Complementary Results for Experiments 1, 2, and 3}

See Tables 6, 7, and 8 .

\section{Disclosure}

The source code is available at GitHub [27].

\section{Competing Interests}

The authors declare no conflict of interests.

\section{Acknowledgments}

This project was supported by the Singapore French Institute (IFS) and the Singapore Eye Research Institute (SERI) through the PHC Merlion Program (2015-2016) and the Regional Council of Burgundy (Grant no. 20159201AAO050S02760). Calculations were performed using HPC resources from DSI-CCUB (Université de Bourgogne).

\section{References}

[1] S. Sharma, A. Oliver-Fernandez, W. Liu, P. Buchholz, and J. Walt, "The impact of diabetic retinopathy on health-related quality of life," Current Opinion in Ophthalmology, vol. 16, no. 3, pp. 155-159, 2005.

[2] S. Wild, G. Roglic, A. Green, R. Sicree, and H. King, "Global prevalence of diabetes: estimates for the year 2000 and projections for 2030," Diabetes Care, vol. 27, no. 5, pp. 1047-1053, 2004.

[3] Early Treatment Diabetic Retinopathy Study Group, "Photocoagulation for diabetic macular edema: early treatment diabetic retinopathy study report no. 1," Archives of Ophthalmology, vol. 103, no. 12, pp. 1796-1806, 1985.

[4] M. R. K. Mookiah, U. R. Acharya, C. K. Chua, C. M. Lim, E. Y. K. Ng, and A. Laude, "Computer-aided diagnosis of diabetic retinopathy: a review," Computers in Biology and Medicine, vol. 43, no. 12, pp. 2136-2155, 2013.

[5] E. Trucco, A. Ruggeri, T. Karnowski et al., "Validating retinal fundus image analysis algorithms: issues and a proposal," Investigative Ophthalmology \& Visual Science, vol. 54, no. 5, pp. 35463559, 2013.

[6] Y. T. Wang, M. Tadarati, Y. Wolfson, S. B. Bressler, and N. M. Bressler, "Comparison of prevalence of diabetic macular edema based on monocular fundus photography vs optical coherence 
tomography," JAMA Ophthalmology, vol. 134, no. 2, pp. 222-228, 2016.

[7] S. J. Chiu, X. T. Li, P. Nicholas, C. A. Toth, J. A. Izatt, and S. Farsiu, "Automatic segmentation of seven retinal layers in SDOCT images congruent with expert manual segmentation," Optics Express, vol. 18, no. 18, pp. 19413-19428, 2010.

[8] R. Kafieh, H. Rabbani, M. D. Abramoff, and M. Sonka, "Intraretinal layer segmentation of 3D optical coherence tomography using coarse grained diffusion map," Medical Image Analysis, vol. 17, no. 8, pp. 907-928, 2013.

[9] P. P. Srinivasan, L. A. Kim, P. S. Mettu et al., "Fully automated detection of diabetic macular edema and dry age-related macular degeneration from optical coherence tomography images," Biomedical Optics Express, vol. 5, no. 10, pp. 3568-3577, 2014.

[10] F. G. Venhuizen, B. van Ginneken, B. Bloemen et al., "Automated age-related macular degeneration classification in OCT using unsupervised feature learning," in Proceedings of the Medical Imaging 2015: Computer-Aided Diagnosis, vol. 941411 of Proceedings of SPIE, Orlando, Fla, USA, February 2015.

[11] Y.-Y. Liu, M. Chen, H. Ishikawa, G. Wollstein, J. S. Schuman, and J. M. Rehg, "Automated macular pathology diagnosis in retinal OCT images using multi-scale spatial pyramid and local binary patterns in texture and shape encoding," Medical Image Analysis, vol. 15, no. 5, pp. 748-759, 2011.

[12] G. Lemaitre, M. Rastgoo, J. Massich, S. Sankar, F. Meriaudeau, and D. Sidibe, "Classification of SD-OCT volumes with LBP: application to DME detection," in Proceedings of the Medical Image Computing and Computer-Assisted Intervention (MICCAI '15), Ophthalmic Medical Image Analysis Workshop (OMIA '15), Munich, Germany, October 2015.

[13] J. Sivic and A. Zisserman, "Video google: a text retrieval approach to object matching in videos," in Proceedings of the 9th IEEE International Conference on Computer Vision (ICCV '03), pp. 1470-1477, Nice, France, October 2003.

[14] J. M. Schmitt, S. H. Xiang, and K. M. Yung, "Speckle in optical coherence tomography," Journal of Biomedical Optics, vol. 4, no. 1, pp. 95-105, 1999.

[15] A. Buades, B. Coll, and J.-M. Morel, "A non-local algorithm for image denoising," in Proceedings of the IEEE Computer Society Conference on Computer Vision and Pattern Recognition (CVPR '05), vol. 2, pp. 60-65, June 2005.

[16] P. Coupe, P. Hellier, C. Kervrann, and C. Barillot, "Nonlocal means-based speckle filtering for ultrasound images," IEEE Transactions on Image Processing, vol. 18, no. 10, pp. 2221-2229, 2009.

[17] T. Ojala, M. Pietikäinen, and T. Mäenpää, "Multiresolution gray-scale and rotation invariant texture classification with local binary patterns," IEEE Transactions on Pattern Analysis and Machine Intelligence, vol. 24, no. 7, pp. 971-987, 2002.

[18] G. Zhao, T. Ahonen, J. Matas, and M. Pietikäinen, "Rotationinvariant image and video description with local binary pattern features," IEEE Transactions on Image Processing, vol. 21, no. 4, pp. 1465-1477, 2012.

[19] D. R. Cox, “The regression analysis of binary sequences," Journal of the Royal Statistical Society. Series B. Methodological, vol. 20, pp. 215-242, 1958.

[20] L. Breiman, "Random forests," Machine Learning, vol. 45, no. 1, pp. 5-32, 2001.

[21] J. H. Friedman, "Stochastic gradient boosting," Computational Statistics \& Data Analysis, vol. 38, no. 4, pp. 367-378, 2002.
[22] G. Lemaitre, J. Massich, R. Martí et al., "A boosting approach for prostate cancer detection using multi-parametric MRI," in Proceedings of the 12th International Conference on Quality Control by Artificial Vision (QCAV '15), Proceedings of SPIE, June 2015.

[23] V. Vapnik and A. J. Lerner, "Generalized portrait method for pattern recognition," Automation and Remote Control, vol. 24, no. 6, pp. 774-780, 1963.

[24] A. Aizerman, E. M. Braverman, and L. I. Rozoner, “Theoretical foundations of the potential function method in pattern recognition learning," Automation and Remote Control, vol. 25, pp. 821-837, 1964.

[25] E. Nowak, F. Jurie, and B. Triggs, "Sampling strategies for bagof-features image classification," in Computer Vision-ECCV 2006, pp. 490-503, Springer, 2006.

[26] D. Arthur and S. Vassilvitskii, "k-means++: the advantages of careful seeding," in Proceedings of the 18th Annual ACM-SIAM Symposium on Discrete Algorithms, pp. 1027-1035, Society for Industrial and Applied Mathematics, New Orleans, La, USA, January 2007.

[27] G. Lemaitre, M. Rastgoo, and J. Massich, "Retinopathy: Joomia-2015," November 2015.

[28] M. K. Garvin, M. D. Abràmoff, X. Wu, S. R. Russell, T. L. Burns, and M. Sonka, "Automated 3-D intraretinal layer segmentation of macular spectral-domain optical coherence tomography images," IEEE Transactions on Medical Imaging, vol. 28, no. 9, pp. 1436-1447, 2009. 


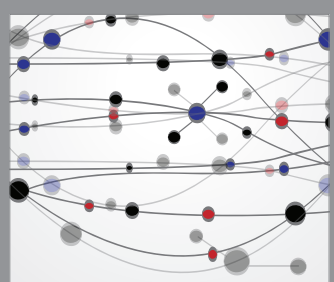

The Scientific World Journal
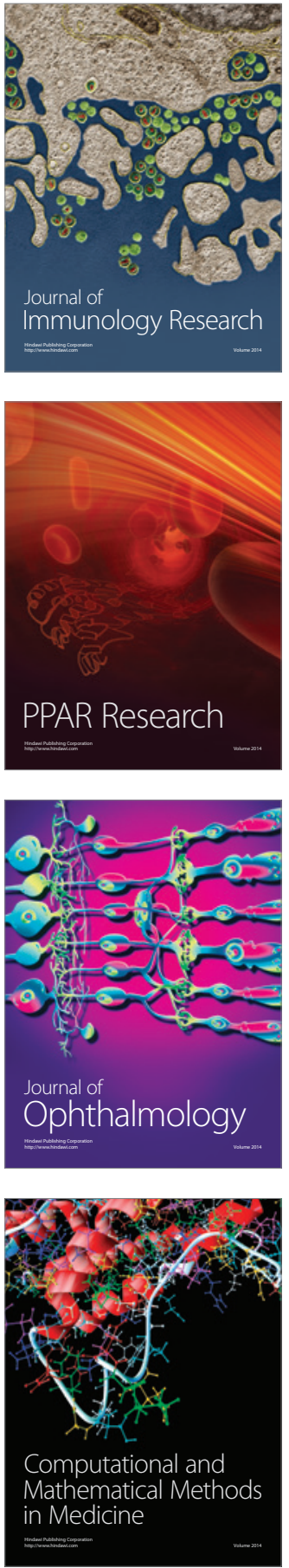

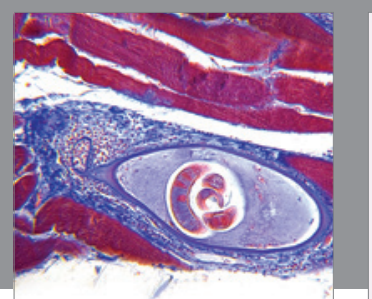

Gastroenterology Research and Practice

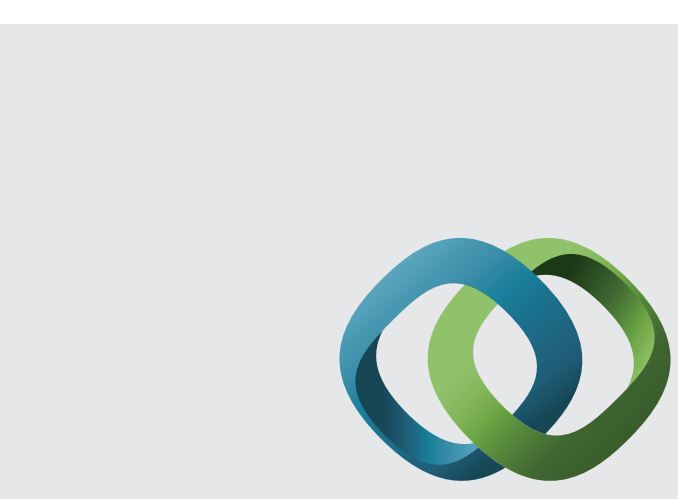

\section{Hindawi}

Submit your manuscripts at

http://www.hindawi.com
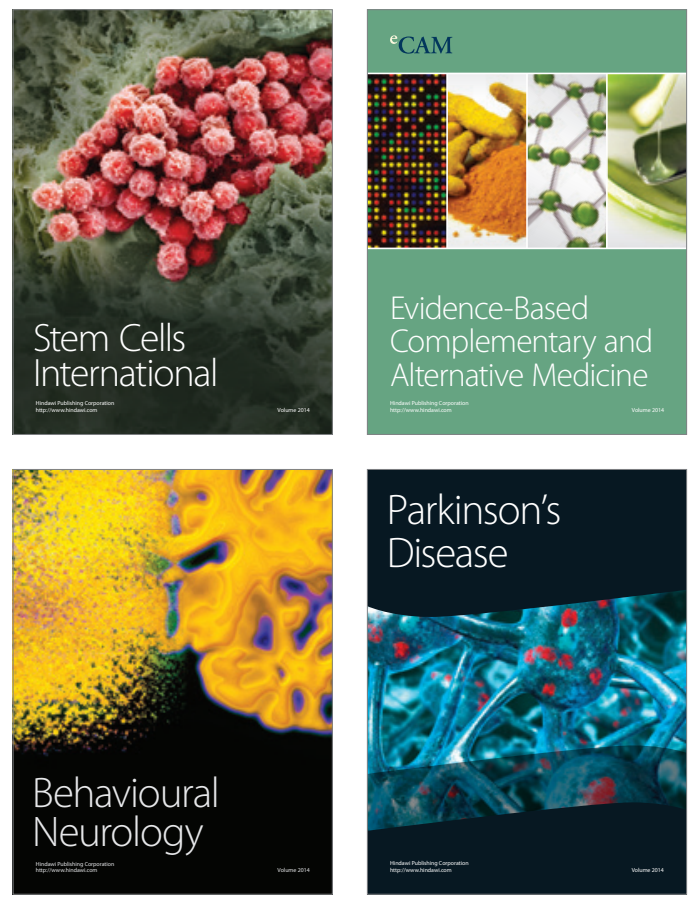
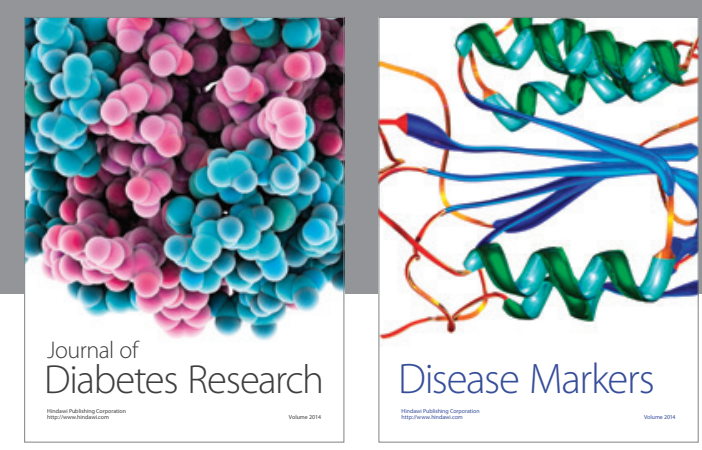

Disease Markers
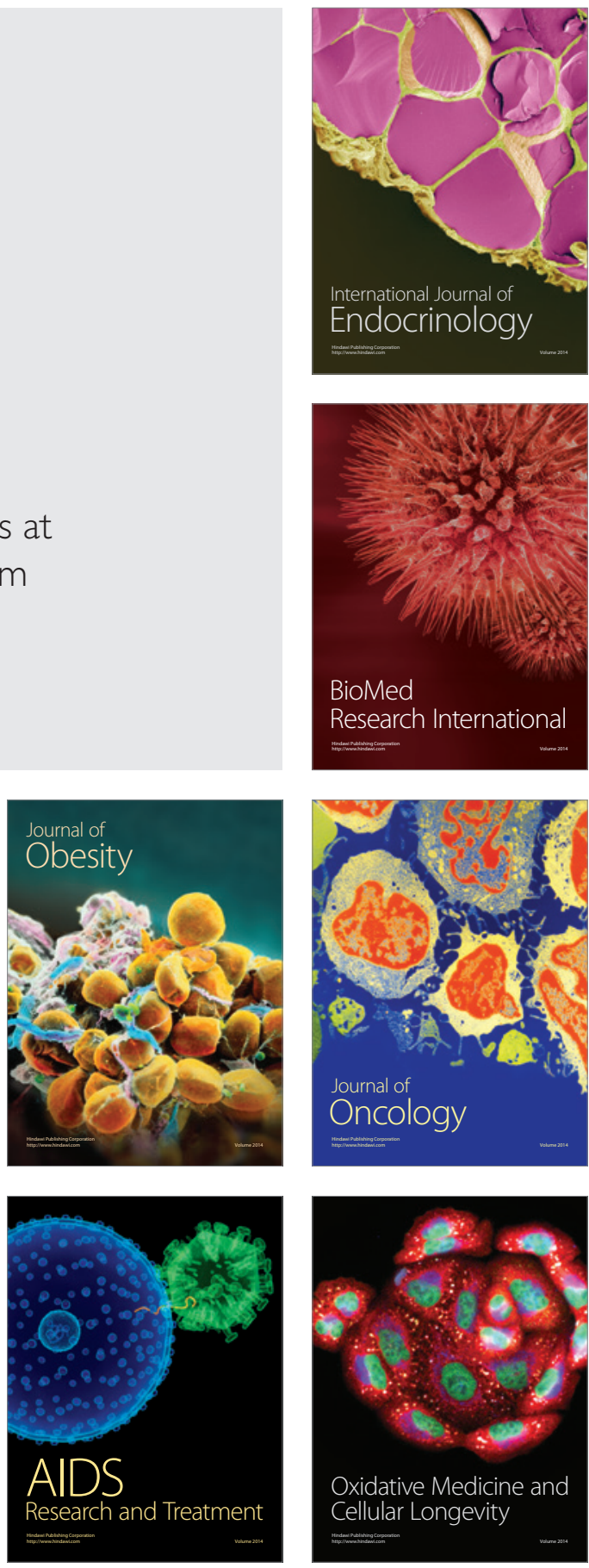